\title{
Numerical study of particle mixing in a lab-scale screw mixer using the discrete element method
}

\author{
Fenglei Qi ${ }^{\mathrm{a}}$, Theodore J. Heindel ${ }^{\mathrm{a}}$, Mark Mba Wright ${ }^{\mathrm{a}, *}$ \\ ${ }^{a}$ Department of Mechnical Engineering, Iowa State University, Ames, Iowa, 50010 USA
}

\begin{abstract}
This study employs the Discrete Element Method (DEM) to simulate particulate flow and investigate mixing performance of a lab-scale double screw mixer. The simulation employs polydispersed biomass and glass bead particles based on experiments conducted in previous studies. Visual examination of particle distribution and statistical analysis of particle residence times of experimental data served as model validation. Statistical analysis indicates a maximum $9.8 \%$ difference between the experimental and simulated biomass particle mean residence time, and visual observations suggest the simulation captures the particle mixing trends observed in the experiments. Results indicate that the particle mean mixing time, non-dimensionalized by ideal flow time in the plug flow reactor, varies between 1.008 and 1.172, and it approaches 1 with increasing biomass feed rate. The mixing index profile in the axial direction shows a mixing-demixing-mixing oscillation pattern. Increasing screw pitch length is detrimental to mixing performance; decreasing the solid particle feed rate reduces the mixing degree; and increasing the biomass to glass bead size ratio decreases mixing performance. A comparison of a binary, single-sized biomass and glass particles mixture to a multicomponent mixture indicates that the binary system has similar mixing pattern as a multicomponent system. These findings demonstrate that DEM is a valuable tool for the design and simulation of double screw mixing systems.
\end{abstract}

Keywords:

Discrete element method; Double screw mixer; Mixing index; Multicomponent mixture; Particle mixing

\section{Introduction}

Screw conveyors/mixers are often used in solid handling processes in industries such as pharmaceutical production, food processing, mineral processing, construction and renewable energy production $[1,2,3]$. Usually single- or double-screws are installed in a housing, and the rotation of the screw $(\mathrm{s})$ results in transportation and mixing of the solid bulk materials.

\footnotetext{
${ }^{*}$ Corresponding author

Email addresses: fqi@iastate.edu (Fenglei Qi), theindel@iastate.edu (Theodore J. Heindel), markmw@iastate.edu (Mark Mba Wright)
}

Preprint submitted to Powder Technology

December 5, 2016 
The merits of handling a wide range of materials, high throughput capacity and relatively narrow residence time distribution of the transported particles [4], make the screw mixer suitable as biomass pretreatment equipment and as a pyrolyzer $[5,6]$ for thermochemically converting biomass into biofuels. Experimental studies have shown comparable bio-oil yield performance of the double screw pyrolyzers when compared to fluidized bed reactors [5].

Achieving a relatively homogeneous particle distribution by mechanical mixing is crucial in a screw mixer in order to guarantee end product quality or enhance other transport process such as heat transfer in screw dryers and pyrolyzers. Particle segregation, which is the opposite process of particle mixing, naturally arises due to any differences in the particle size, shape, density or other particle properties especially when mechanical agitation is provided to the bed. Current design of mechanical mixers often depends on empirical correlations or a priori knowledge. For example, in the design of a pyrolyzer [7], a double screw design was adopted over a single screw based on engineering knowledge. The optimal operating conditions have to be repeatedly tested due to limited information regarding mixing performance of the screw pyrolyzer in terms of screw geometry and operating conditions $[5,8]$. Quantitatively assessing the mixing performance of the screw mixer is necessary for successful equipment design or scale-up .

Solid particle mixing processes have been analyzed based on several experimental approaches such as sampling [8,9], X-ray tracking [10, 11], positron emission particle tracking (PEPT) [12] and digitized image analysis methods [13]. Tsai and Lin [9] qualitatively studied the effects of screw parameters (pitch, flight diameter, flight thickness and shaft diameter) and operating conditions (rotation speed) on the mixing quality in a single screw feeder. Their sampling and analysis method was updated by [14] for qualitatively analyzing the mixing degree in a screw dryer. Kingston and Heindel [8] analyzed sample homogeneity obtained from four different ports at the outlet, and the variance of the sampling was employed to characterize the mixing quality in a double screw mixer. The sampling methods reported in $[8,9,14]$ can only assess the overall mixing quality in the screw mixer, and some results from their analysis yield contradictory conclusions. For example, the particle mixing degree was reported to decrease with increasing pitch in [9] while it is reported to increase in [8]. More advanced particle tracking techniques such as PEPT have shown great potential in characterizing particle flow pattern and quantifying mixing quality [12]. However, tracers used in the techniques must be developed to meet the specifications of the studied particle system. Segregation of tracer particles from bulk particles occurs as a consequence of density or size differences [15]. Besides, the more advanced tracking techniques are expensive and time consuming for conducting parametric studies of mixer systems.

As an alternative approach, the discrete element method (DEM) is often employed to perform numerical simulations of particle dynamics. This method was first introduced by Cundall and Strack [16]. Particle movement, including particle position and velocity, is numerically computed based on Newton's equations of motion which considers driving forces such as contact, cohesion and drag forces exerted by the fluid phase and other particles. The advantage of DEM is that particle motion is directly resolved and eliminates the need for a rheological model in order to solve the momentum of particles as in two fluid models (TFM) [17]. The limitation of relatively high computational costs of DEM can be overcome 
by adopting parallel computing techniques [18] or by introducing coarse-grain models [19]. Some demonstrations of discrete element simulation of industrial particulate systems are found in $[20]$.

DEM has been widely used to analyze bulk material transportation characteristics, flow patterns and mixing processes in screw conveyors/mixers as well as other mixer types. Tanida et al. [21] and Shimizu and Cundall [12] employed DEM to simulate granular flow and mixing in screw conveyors. Schutyser et al. [22] compared predictions from a DEM model to experimental measurements using PEPT in the study of particulate flow and mixing in a conical helical-blade mixer. They observed a good match between the model predictions and the experiments. The mixing mechanism for this particular device was also analyzed and quantified. Further validation of DEM in modeling continuous mixers was studied in $[15,23]$, which boosts the adoption of DEM in quantitatively analyzing mixing processes in various continuous mixers such as the plowshare mixer [24], twin-screw kneader [25], ribbon mixer [26], and convective continuous mixer [27, 28]. Owen and Cleary [29, 30] applied DEM to simulate a single screw conveyor and examined the influence of operating conditions on the power consumption and feeding performance of the device. Similar research was also carried out in [31]. They compared DEM predictions of mass flow rate and experimental measurements, and observed consistency between modeling results and experiments. Pezo et al. [32] analyzed the particle pathways in a single screw conveyor-mixer with various screw designs, and they showed that adding blades to the screw could generally increase particle path length and achieve auxiliary mixing performance. Hou et al. [33] considered the effects of cohesion forces on the particle flow dynamics in a screw feeder. Although DEM has shown a lot of potential in screw conveyor/mixer design and particulate system hydrodynamic analysis, most of the previous studies are limited to monodispersed or monocomponent particle systems. Understanding of screw mixers for polydispersed or multicomponent particle systems is still lacking. Additionally, few studies have been done to examine the mixing process in the double screw mixer.

Particle size distribution commonly exists in particulate systems and properly considering the particle size distribution is necessary to accurately replicate the granular flow dynamics in the numerical simulation. In this research, DEM was applied to investigate particulate flow and mixing in a lab-scale double screw mixer in which polydispersed biomass particles and glass beads are transported and mixed. The DEM method was first introduced and a mixing index evaluating the mixing state of the multicomponent mixture was developed in the next section. The DEM model was then validated by comparing DEM predictions with experimental studies [34] through visual examination of particle distribution and particle residence time comparisons. Screw pitch length, screw rotation speed and biomass feed rate were varied, and the effects of the operating conditions on the mixing performance of the double screw mixer were quantitatively examined. The mixing performance of particulate systems with different size ratios of biomass to glass beads was also examined. 


\section{Model description}

\subsection{Model system}

The geometry of the modeled double screw mixer is shown in Figure 1. Two screws are installed in the trough. The left screw flight is left-handed and the right screw flight is right-handed. As show in the Figure 1a, when the left screw is rotating in the clockwise direction and the right screw is rotating in the counter-clockwise direction, the bulk material is pushed forward from the inlet side to the outlet side along the axial direction. At the cross section, the materials are pumped downwards to the trough wall at the center of the mixer. This material flow pattern in the double screw mixer is commonly called "Counter-Rotating Down Pumping" [8]. The screw rotation speed varied from 20 to 60 RPM in this study.

Major dimensions of the mixer are mixing length $L$, mixer trough width $W$, mixer trough height $H$, and shaft center distance $S$. The curvature of the bottom part of the trough can be calculated from the trough width and the center distance between the two shafts. Major dimensions of the installed screws are flight diameter $D$, shaft diameter $d$ and flight pitch $P$. The gap between the trough bottom and screw flight can be calculated from the flight diameter and trough dimensions and is usually kept less than the particle diameter. All the dimensions remained constant except the flight pitch in this study. The effects of pitch length on the mixing performance were investigated.

There are two inlets which separately feed biomass material (red oak) in inlet 1 and glass beads in inlet 2 into the mixer as shown in Figure 1a. The biomass particle size in the experimental study [8] varied from $500 \mu \mathrm{m}$ to $6350 \mu \mathrm{m}$ and glass beads had a particle size range of 300 to $500 \mu \mathrm{m}$. In the simulations, a spherical particle shape was employed to represent both biomass and glass particles. A mass-based uniform distribution of biomass particles with the diameter of $1.0 \mathrm{~mm}, 1.5 \mathrm{~mm}, 2.5 \mathrm{~mm}, 3.5 \mathrm{~mm}, 4.5 \mathrm{~mm}$ and $5.5 \mathrm{~mm}$ were modeled as biomass feedstock, and a monosized particle of diameter $1.0 \mathrm{~mm}$ was adopted for glass bead particles. The mass feed ratio of biomass to glass beads was fixed to 1:10 in all of the studies based on common experimental conditions. Different volumetric fill levels were achieved by changing the material feed rate. In order to avoid the effects of feed frequency on the analysis, the feed frequency for both biomass and glass beads was scaled accordingly in terms of the biomass feed rate whenever the screw rotation speed, pitch length or biomass feed rate was changed.

The three parameters, screw rotation speed, flight pitch and biomass feed rate, were varied in the numerical experiments. Table 1 shows the simulation cases that were performed in order to investigate the effects of the three parameters on the mixing process in the mixer. In the table, case 1-1, 1-2 and 1-3 are designed for a screw rotation speed study; case 2-1, 2-2 and 2-3 for a pitch length study; and case 3-1, 3-2 and 3-3 for a solid mass feed rate study. The solid mass feed rate was adjusted when the rotation speed or the pitch length was changed in order to keep the same volumetric fill level and 1:10 biomass-to-glass bead mass flow ratio. Two binary systems (case 4-1 and 4-2) were also modeled and compared to the multicomponent system. In case 1-2, an average number of 14200 biomass particles remains in the mixer during the simulation, and an average number of 101400 glass beads is simultaneously modeled in the mixer. The number of modeled particles in case 1-1, 1-3, 2-1 
and 2-3 are close to that in case 1-2 since a constant volumetric fill level is remained. The average number of modeled particles in case 3-1 is approximately 5400 of biomass particles and 34100 of glass beads. The average number of particles in case 3-2 is approximately 9100 of biomass particles and 60000 of glass beads.

\subsection{Discrete element method (DEM)}

The discrete element method (DEM) is a numerical technique that resolves each individual particle motion in a particulate system that contains a collection of particles. Heat transfer and chemical reactions can also be resolved at the particle scale with advanced particle-scale models $[35,36]$ which extend the capabilities of DEM. With regard to particle dynamics, the DEM model in this work is based on the so-called soft sphere approach that has been implemented in LIGGGHTS [37]. In this approach, the particles are allowed to overlap at collision and the contact forces are subsequently calculated from the local deformation history at the contact point using various force-displacement laws such as the linear spring-dashpot model and the Hertz-Mindlin nonlinear model [38]. In this research, the Hertz-Mindlin model in LIGGGTHS was employed which is based on the original approach proposed in $[39,40]$. The translational and rotational motion are resolved by numerically integrating Newton's and Euler's equations for a spherical shape particle, written as

$$
\begin{aligned}
m_{i} \frac{d \boldsymbol{v}_{i}}{d t} & =\sum_{j} \boldsymbol{F}_{i j}^{c}+\boldsymbol{F}_{i}^{f}+\boldsymbol{F}_{i}^{g}, \\
I_{i} \frac{d \boldsymbol{w}_{i}}{d t} & =\sum_{j}\left(\boldsymbol{T}_{t, i j}+\boldsymbol{T}_{r, i j}\right),
\end{aligned}
$$

where, $m_{i}$ and $I_{i}\left(=2 / 5 m_{i} R_{i}^{2}\right)$ are the mass of particle $i$ and the moment of inertia of the particle, respectively. $\boldsymbol{v}_{i}$ and $\boldsymbol{w}_{i}$ are the translational and rotational velocities of the particle. The forces in Equation (1) include the contact force $\boldsymbol{F}_{i j}^{c}$ between particle $i$ and surrounding particle $j$ that collides with particle $i$, possible drag forces acting on particle $i$ by a fluid $\boldsymbol{F}_{i}^{f}$, and the gravitational force $\boldsymbol{F}_{i}^{g}$. The torques acting on particle $i$ includes both $\boldsymbol{T}_{t, i j}$ and $\boldsymbol{T}_{r, i j}$. Torque $\boldsymbol{T}_{t, i j}$ is generated by tangential force $\boldsymbol{F}_{t, i j}^{c}$ which is the component of the contact force $\boldsymbol{F}_{i j}^{c}$ with direction parallel to the contacting surface between particle $i$ and $j$. Torque $\boldsymbol{T}_{r, i j}$, called rolling friction torque, is generated by the asymmetric distribution of the normal contact force $\boldsymbol{F}_{n, i j}^{c}$, the other component of $\boldsymbol{F}_{i j}^{c}$ with direction perpendicular to the contact surface. In this research, the drag forces are ignored in the bed of the screw mixer since the major forces arise from mechanical torques by the shaft. The mechanical forces are transferred by particle-particle contact/collision and particle-wall contact/collision in the bed. The equations for calculating forces and torques acting on particle $i$ are summarized in Table 2.

In order to detect collisions between particles and complicated mixer walls, the mixer surfaces are discretized into triangle meshes. For example, 6816 triangle meshes are employed to represent each shaft (Figure 1) and the average surface area of the triangle mesh is equivalent to the surface area of a sphere with radius of $0.375 \mathrm{~mm} ; 6836$ triangle meshes are 
used for the trough and the average surface area of the triangle mesh is equivalent to the surface area of a sphere with radius of $0.72 \mathrm{~mm} ; 7494$ triangle meshes are used to represent each screw with pitch length $P / D=1.25$ and the average triangle mesh area is equivalent to the surface area of a sphere with radius of $0.464 \mathrm{~mm}$. The surface discretization scheme restores the surface curvature very well and shows little effects on the simulation results. The contact force calculation between the spherical particle and triangle mesh is similar to that between sphere and sphere particles. The radius and mass of the triangle mesh can be set to a large number when using the equations shown in Table 2 . The rotation of the screws and shafts can also be accomplished in LIGGGHTS [37].

Material inertial and mechanical properties in the DEM models are listed in Table 3. A smaller material Young's Modulus was used as a general practice in order to reduce computational time [41]. Friction coefficients between different materials were set to the same value which was calibrated in terms of biomass particle mean residence time by comparing to experimental studies [34] at a single operating condition (case 1-2 listed in Table 1). The rolling friction coefficient was kept the same for all materials as well.

\subsection{Mixing index}

In this system, both particle size differences among biomass particles and density differences between biomass and glass beads exist, and particle segregation is the result of these combined effects. We identify particle segregation incurred by material density difference is more detrimental to the mixing goal. In order to quantify the mixing degree between biomass and glass beads, a pseudo binary mixture was considered in which one component is glass beads and the other is biomass. The mixing index [42] is formulated as:

$$
M=1-\frac{\sigma_{t}^{2}}{\sigma_{0}^{2}}
$$

where, $\sigma_{t}^{2}$ and $\sigma_{0}^{2}$ are the composition variance in a mixture and composition variance in a fully segregated mixture, separately. They are calculated by

$$
\begin{gathered}
\sigma_{t}^{2}=\sum_{i=1}^{N} w_{i}\left(x_{i}-x_{m}\right)^{2}, \\
\sigma_{0}^{2}=\frac{\rho}{\rho_{g}}(1-p)^{2} p+p^{2}\left(1-\frac{\rho}{\rho_{g}} p\right),
\end{gathered}
$$

where, $N$ is the number of sampling cells. $x_{i}$ represents the glass bead mass fraction in a cell as shown in Figure 2. $x_{m}$ is the average glass bead mass fraction in all sampling cells. $w_{i}$ is a weight factor accounting for the different sampling size effects and is calculated as the total solid volume in a cell divided by the total solid volume in all sampling cells [41]. $p$ is the overall mass flow ratio of glass beads to total solid mass flow rate. $\rho_{s}$ is the glass bead true density and $\rho$ is the mean true density of glass and biomass mixture calculated as

$$
\frac{1}{\rho}=\frac{p}{\rho_{s}}+\frac{1-p}{\rho_{b}}
$$


where, $\rho_{b}$ is the biomass true density. A large composition difference among samples results in a large value of $\sigma_{t}^{2}$. $\sigma_{t}^{2}$ achieves the upper limit value of $\sigma_{0}^{2}$ when the mixture is fully segregated. On the contrary, $\sigma_{t}^{2}$ achieves the lower limit value of 0.0 when the mixture composition is uniform. The resulting range of mixing index $M$ is $[0,1]$.

As shown in Figure 2, a grid system was used for different sample locations. The grid has 3 cells per pitch in the axial direction for $\mathrm{P} / \mathrm{D}=0.75,5$ for $\mathrm{P} / \mathrm{D}=1.25$ and 7 for $\mathrm{P} / \mathrm{D}=1.75$. The number of cells in the transverse section is $4 \times 8$. Considering the symmetry of the geometry and flow, only the right part of the grid in the transverse section was used for the sampling and mixing analysis. The size of the mesh was determined so that the average particle number in the cells is between 30-150. This is because the variance $\sigma_{t}^{2}$ is inaccurate if a cell is too small [43]. In the sampling grid system, the average number of particles is approximately 80 per sampling cell. In order to guarantee each sample size, a sampling cell in which the solid fraction is less than 0.2 is not considered as effective sample. This limit prevents the cells containing a smaller amount of particles to be included in the mixing analysis.

\section{Results and Discussion}

\subsection{Model validation}

The DEM model was validated by comparing simulated results to experimental data reported in [34]. The dimensions of the mixer and operating conditions in the simulation were identical to the experimental studies. Visual comparison of granular flows in the double screw mixer is shown in Figure 3. Snapshots from the top view and right view were taken in the experimental studies and DEM simulations. The operating conditions are shown in Table 1 in case 1-2. In the experimental snapshots, the biomass (red oak) particles have a natural brown color and the glass beads are gray. In order to distinguish biomass particles from glass beads in the simulations, blue is used to render biomass particles and red is used for simulated glass beads as seen in the snapshots taken from the DEM results.

Particle distribution observed in the experimental study was qualitatively predicted in the DEM simulation based on visual examination of the snapshots from both the experimental study and DEM simulation. The smaller and heavier glass particles are partially separated from the biomass particles and sink to the bottom of the mixer covering the bottom part of the trough. The lighter and larger biomass particles are barely observed near the trough wall from the right view in both the experimental and DEM snapshots, while the biomass particles are mostly observed from the top view in both the experimental and DEM snapshots. The differences from visual observations of the volumetric fill level in the experimental mixer and simulated mixer are negligible. The back flow of glass particles observed in both experiments and DEM simulations is shown in the box in the right view. It is also observed from both the experiments and DEM simulations that a discharging slope is formed near the outlet.

The DEM model was further validated by comparing with the experimental results $[11,34]$ in terms of biomass mean residence time at different screw rotation speeds as shown in Figure 4. The corresponding operating conditions are listed in case 1-1, 1-2 and 1-3 in Table 1. The experimental data consists of three measurements for each operating condition 
and some data points overlapped with each other. When calculating the mean residence time from the DEM results, biomass particles continuously fed into the mixer in a given period were tracked until over $95 \%$ percent of the tracked particles left the simulation domain. The mean residence time was averaged over the tracked particles that left the domain, and the standard deviation of particle residence time was also calculated. From Figure 4, it is observed that the predicted biomass mean residence time is in good agreement with experimental observations. All the experimental data points are within the standard deviation range. Statistical analysis indicates a maximum $9.8 \%$ difference between the experimental and simulated biomass particle mean residence times. This implies that the DEM models is capable of predicting biomass and glass bead mixture flow durations in the double screw mixer. It is also observed that the standard deviation range is reduced when the screw rotation speed increases.

\subsection{Mixing time}

The mixing time is an indication of how long the two materials are mixed in the double screw mixer. This is an important factor when designing a double screw reactor such as a pyrolyzer. The mixing time determines whether biomass particles reside long enough in the equipment to allow desired processes to occur, such as particle heating and chemical reactions.

The mean mixing time corresponds to the average particle duration in the mixing length $L$ shown in Figure 1. The mixing time of a biomass particle is equal to the particle residence time minus the time spent from inlet 1 to inlet 2 (Figure 1). The same tracking technique was used to obtain particle mixing time from the DEM simulations as for calculating particle residence time.

The mean mixing time of the biomass particles is plotted in Figure 5. The time was non-dimensionalized in terms of the flow time in the ideal plug flow reactor with the same operating condition. The non-dimensional particle mean mixing time varies between 1.008 and 1.172, and it approaches 1.0 when the biomass feed rate is increased. This observation indicates that the major movement of particles is in the axial direction in the screw rotation speed range of 20-60 RPM. The pathlines of two random particles are illustrated in Figure 6 . It is noted that increasing the rotation speed increases the non-dimensional mean mixing time, and the increment is nonlinear in terms of the rotation speed. This observation is in agreement with the cited experimental study [34]. Additionally, a larger pitch length could increase the non-dimensional mean mixing time, and the increment is also nonlinear in terms of pitch length. The confidence interval becomes wider as the pitch length increases. When adopting a larger pitch length screw, the axial velocity of particles is increased which has similar effects on the mixing time as adopting higher screw rotation speed. The mean mixing time increases when the biomass feed rate is reduced. One explanation is that the gap between the screw flight and trough wall starts to influence the biomass movement at low biomass feed rates. 


\subsection{Visual observations of particle mixing in the double screw mixer}

The effects of screw rotation speed, pitch length and biomass feed rate on the mixing process in the double screw mixer were investigated. The counter-rotating down-pumping pattern of the double screws generates a slope in the bed from the trough wall to the mixer center at the transverse section of the mixer. The slope can be observed in Figures 7-10. Some particles slide down the slope and are later pushed up the slope forming a recirculation in the transverse section. This recirculation can be observed by following the pathline of a single particle as shown in Figure 6. Although this recirculation is much weaker than the recirculation observed in the rotary drum mixer [44], it may have the same function for promoting particle mixing.

A cross section image of the mixer (slice thickness: $6 \mathrm{~mm}$ ) was gathered to visually observe the particle distribution within the mixer. The image location is at $L_{m}=3.75 D$, which is three pitch lengths downstream of the glass feed inlet, and $P / D=1.25$.

Figure 7 shows the particle distribution within the slice at different screw rotation speeds. The volumetric fill level, bed slope and particle distribution are very similar over the investigated rotation speed range, which indicates the screw rotation speed has negligible effects on the mixing process.

Figure 8 compares particle distribution in a full rotation period at the slice when different pitch lengths are adopted for the mixer. The differences in the particle distribution are not discernible from visual examination when the ratio of pitch length to the flight diameter $(P / D)$ changes from 0.75 to 1.25 . However, the change in the particle distribution is obvious when this ratio varies from 1.25 to 1.75 . When the larger pitch length $(P / D=1.75)$ is employed, the particle bed on the trailing side of the flight is lower in the first two phases of the period compared to that when $P / D=1.25$. The particle distribution is less uniform during the rotation period with $P / D=1.75$. The bed slope in the axial direction was further observed from the particle distribution in the longitudinal section as shown in Figure 9. The bed slope increases gradually when the pitch length increases. The change in the bed slope is relatively mild from $P / D=0.75$ to $P / D=1.25$ compared to the change from $P / D=1.25$ to $P / D=1.75$. At $P / D=1.75$, a large and long distance slope is observed. The formed slope could enhance the sliding of lighter biomass particles and incur the segregation of biomass particles from the glass beads which is undesirable for mixing processes. Although the screw rotation could generate another recirculation in the longitudinal section as reported in [14], the formation of a large slope is more favorable to segregation than mixing as suggested by the particle distribution. Furthermore, the free space near the trailing edge of the flight seen in Figure 9(c) could also allow for biomass particles to float up to the surface from the mixture due to the density differences, which incurs particle segregation in the transverse section. Although the formation of the slope was also observed in the experimental study at $P / D=1.75$ [34], the sampling analysis reported in [8] indicated increasing the screw pitch enhanced particle mixing, which is opposite to current observations of DEM simulation results. On the DEM simulation side, the contradictory observations might be due to the biomass particle sphericity. The simulation biomass particles are perfect spheres and exhibit greater propensity to roll on surfaces and against other particles. Experiments indicate that 
the non-spherical shape of real biomass particles allows auger screws to lift biomass particles, which affects the granular flow mixing pattern. This effect was not investigated in this study.

Figure 10 illustrates the particle distribution at different biomass feed rates while the biomass-to-glass bead mass flow rate is maintained at 1:10. The volumetric fill level in the auger mixer is reduced when the biomass feed rate decreases. At the biomass feed rate of $f=3.5 \mathrm{~kg} / \mathrm{h}$, some particles are lifted up by the screw flight and run across the shaft into the other side, as shown in the last column of Figure 10. This flow pattern contributes to particle convective mixing. However, this flow pattern disappears at biomass feed rates of 1.0 and $2.0 \mathrm{~kg} / \mathrm{h}$. Under these conditions, the free space under the shaft would also allow for the biomass particles to segregate from the mixture bed in the transverse section.

\subsection{Mixing state evaluation in the double screw mixer}

The mixing index discussed in Section 2.3 was employed to quantitatively evaluate the mixing state of the multicomponent mixture. The biomass particles were considered as one component and a pseudo binary mixture system of biomass and glass beads was assumed in calculating the mixing index. Each plane of the grid in the transverse direction shown in Figure 2 was considered as a section and each cell in the section was considered as one sampling element. Instantaneous sampling was made in all sections 20 times equally in a period for five periods after the flow achieved steady state. The mixing index was calculated for the plane in terms of the composition variances in all sampling cells in a section. The mixing index along the axial direction is shown in Figure 11.

Some common features are observed for all cases. At the beginning, there are only biomass particles in the first few planes and the variance of the composition is zero. According to the definition of the mixing index in this study, this corresponds to a mixing index of 1. However, this does not indicate a well mixed system of biomass particles and glass beads since only biomass particles exist at these planes. Due to the back flow of glass beads shown in Figure 3, the mixing starts before the glass bead feed inlet and a relatively small local mixing index is seen before the feed location. The value of the mixing index increases in the next several planes and then oscillates around a value in a regular pattern. The index oscillation as visually seen in Figures 7,8 and 10 indicates varying mixing and segregation states along the axial direction. As particles are transported downstream, particle axial segregation occurs in a regular mixing-demixing-mixing pattern. In most cases, the mixing index at the exit section is small because of particle segregation at that point.

The first row in Figure 11 shows the mixing index profiles along the axial direction at different screw rotation speeds. The mixing index profiles are very similar in terms of index value, oscillation amplitude and pattern. The index value varies in a wide range from 0.6 to 0.8 with similar pattern after achieving steady oscillation. At higher rotation speeds (60 RPM), more kinematic energy is provided and the maximum value of the mixing index decreases along axial direction, which indicates particle segregation is enhanced in the downstream. The oscillation period of the mixing index in the axial direction is approximately consistent with the screw pitch.

The second row in Figure 11 shows the mixing index profiles at different pitch lengths. The mixing-demixing-mixing pattern remains when the pitch length is changed but the 
mixing index profile varies in terms of the index value, oscillation amplitude and frequency. The mean value of the mixing index decreases when the pitch length is increased. The approximate mean value is 0.83 for $P / D=0.75,0.75$ for $P / D=1.25$, and 0.6 for $P / D=$ 1.75. The oscillation amplitude is enhanced as the pitch length is increased which indicates axial segregation is enhanced and an inferior mixing is expected. Besides, the oscillation frequency varies when the pitch length is changed. The oscillation period of the mixing index is approximately consistent with the screw pitch in each case.

It is worth noting that the simulation result of the effects of pitch length on the mixing degree is not consistent with that reported by Kingston and Heindel [8]. In addition to the effects of particle shape in DEM simulations and sampling method in the experiment on the concluded results, the sampling location could have a great impact on the mixing performance evaluation of the double screw mixers. Sampling at a single location may not reflect the mixing-demixing-mixing pattern in the double screw mixers observed from the DEM simulation results.

The third row in Figure 11 shows the mixing index profiles at different biomass feed rates. It is observed that reducing biomass feed rate significantly enhances the oscillation amplitude, which indicates axial segregation is enhanced at a lower biomass feed rate. The oscillation period of the mixing index is consistent with the screw pitch.

The quantitative analysis of the mixing index at each plane is in agreement with the analysis from the visual observations of DEM simulations. Generally, screw rotation speed does not affect the mixing process in the double screw mixer significantly, which is consistent with Kingston and Heindel [8], but the transport capacity increases significantly with higher rotation speed as shown in Table 1. The particle segregation in the axial direction is enhanced when the pitch length increases. Reducing biomass feed rate is not favorable for particle mixing.

\subsection{Mixture composition effects on mixing state}

Three mixture systems (case 1-2, 4-1 and 4-2 in Table 1) were also investigated to determine how the mixture system affects the performance of the double screw mixer under the same operating conditions. Figure 12 shows snapshots of the particle distribution at location $L_{m}=3.75 \mathrm{D}$ from the glass bead feed inlet in three mixture systems. The particles have a similar distribution in three mixture systems and approximately the same volumetric fill level. It was also observed a better particle mixing in binary mixture I which is composed of monosized biomass and glass particles compared to mixture system II and multicomponent mixture systems, which indicates increasing size ratio of biomass particles to glass beads is not beneficial to the particle mixing.

Figure 13 shows the mixing index at each plane. Quantitative analysis shows differences in the mixing index profiles along the axial direction in terms of the index mean value and oscillation amplitude. In the binary mixture of monosized biomass and glass particles (Figure 13 (a)), a higher mixing degree is achieved than the other two mixture systems, which is consistent with the visual observations, and the mixing index oscillation along the axial direction is negligible. The mixing index profiles have some similarities between the binary mixture of glass beads and biomass particles with diameters of 1 and $2 \mathrm{~mm}$ (Figure 
13 (b)), and the multicomponent mixture (Figure 13 (c)). The two mixture systems have similar mixing-demixing-mixing pattern and the mean mixing index value is approximately the same. The oscillation period of the mixing index profile in both systems is consistent with the screw pitch. However, a smaller oscillation amplitude is observed for the binary mixture of glass beads and biomass particles with diameters of 1 and $2 \mathrm{~mm}$ compared to the multicomponent mixture.

\section{Conclusion}

The current research presented a DEM study of the particulate flow in a lab-scale double screw mixer in which polydispersed biomass particles and glass beads are transported and mixed. The biomass particle size distribution was considered in the DEM model. We found that a mixing-demixing-mixing oscillation pattern exists along the axial direction in the mixer for all the multicomponent mixture systems, which is not observed in a monosized mixture system. Compared to experimental research, DEM provides more insights of the granular flow in the mixer such as particle trajectory, particle velocity, as well as local mixing state, which are either economically expensive or impossible to be measured with current experimental techniques.

The model was validated by comparing the DEM predictions with experimental studies through visual examination of particle distribution and statistical analysis of particle residence time. Statistical analysis indicates a maximum $9.8 \%$ difference between experimental and simulated biomass particle mean residence time, and visual observations suggest the simulation captures the particle mixing trends found in the experiments.

Screw rotation speed, screw pitch length and solid particle feed rate were varied and the effects on the mixing performance of screw mixer were quantitatively evaluated in terms of mean particle mixing time and mixing index in the multicomponent mixture system. The particle mean mixing time non-dimensionalized by the flow time in the ideal plug flow reactor varies between 1.008 and 1.172, and it approaches 1.0 when the biomass feed rate is increased. Increasing screw rotation speed in a range of 20-60 RPM or screw pitch length increases the average particle mixing time to a limited degree. Reducing biomass feed rate increases mean mixing time due to the influence of gap between the screw flight and trough wall.

Screw rotation speed has negligible impact on the mixing degree in the screw mixer when the volumetric fill level and screw pitch remained constant. Increasing the screw pitch length is detrimental to the mixing performance due to enhanced particle axial segregation. Decreasing the solid particle feed rate results in a decline in the mixing degree, and segregation in the transverse section is increased as a possible result of free space under the shaft.

Mixing performance of the screw mixer for three different mixture systems were compared. Increasing the ratio of biomass size to the glass bead size results in inferior mixing. The mixing pattern of the binary mixture of glass and biomass beads with diameters of 1 and $2 \mathrm{~mm}$ is similar to the multicomponent mixture system which indicates a possibility that the binary mixture system can be modeled as a surrogate of the multicomponent system in engineering applications. 


\section{Acknowledgements}

The research reported in this paper is partially supported by the HPC@ISU equipment at Iowa State University, some of which has been purchased through funding provided by NSF under MRI grant number CNS 1229081 and CRI grant number 1205413.

Funding: This work was supported by the National Science Foundation EPSCoR program [Grant EPS-1101284]. 


\section{References}

[1] S. Waje, B. Thorat, A. Mujumdar, Screw conveyor dryer: Process and equipment design, Drying Technology 25 (1) (2007) 241-247.

[2] J. Dai, J. R. Grace, A model for biomass screw feeding, Powder Technology 186 (1) (2008) 40-55.

[3] R. M. Dhenge, K. Washino, J. J. Cartwright, M. J. Hounslow, A. D. Salman, Twin screw granulation using conveying screws: Effects of viscosity of granulation liquids and flow of powders, Powder Technology 238 (2013) 77-90.

[4] R. Nachenius, T. van de Wardt, F. Ronsse, W. Prins, Torrefaction of pine in a bench-scale screw conveyor reactor, Biomass and Bioenergy 79 (2015) 96-104.

[5] J. Brown, R. Brown, Process optimization of an auger pyrolyzer with heat carrier using response surface methodology, Bioresource Technology 103 (1) (2012) 405-414.

[6] S. Kelkar, C. M. Saffron, L. Chai, J. Bovee, T. R. Stuecken, M. Garedew, Z. Li, R. M. Kriegel, Pyrolysis of spent coffee grounds using a screw-conveyor reactor, Fuel Processing Technology 137 (2015) 170-178.

[7] J. N. Brown, Development of a lab-scale auger reactor for biomass fast pyrolysis and process optimization using response surface methodology, Master's thesis, Iowa State University, Ames, Iowa, USA (2009).

[8] T. A. Kingston, T. J. Heindel, Granular mixing optimization and the influence of operating conditions in a double screw mixer, Powder Technology 266 (2014) 144-155.

[9] W.-R. Tsai, C.-I. Lin, On the mixing of granular materials in a screw feeder, Powder Technology 80 (2) (1994) 119-126.

[10] T. A. Kingston, T. B. Morgan, T. A. Geick, T. R. Robinson, T. J. Heindel, A cone-beam compensated back-projection algorithm for X-ray particle tracking velocimetry, Flow Measurement and Instrumentation 39 (2014) 64-75.

[11] T. A. Kingston, T. A. Geick, T. R. Robinson, T. J. Heindel, Characterizing 3D granular flow structures in a double screw mixer using X-ray particle tracking velocimetry, Powder Technology 278 (2015) 211-222.

[12] Y. Shimizu, P. A. Cundall, Three-dimensional DEM simulations of bulk handling by screw conveyors, Journal of Engineering Mechanics 127 (9) (2001) 864-872.

[13] C. Huo, P. Feng, C. Fan, W. Lin, W. Song, Characterization of transverse mixing in a screw mixer by image analysis, Drying Technology 34 (2) (2016) 194-205.

[14] S. Waje, B. Thorat, A. Mujumdar, Hydrodynamic characteristics of a pilot-scale screw conveyor dryer, Drying Technology 25 (4) (2007) 609-616.

[15] B. Laurent, P. Cleary, Comparative study by PEPT and DEM for flow and mixing in a ploughshare mixer, Powder Technology 228 (2012) 171-186.

[16] P. A. Cundall, O. D. Strack, A discrete numerical model for granular assemblies, Geotechnique 29 (1) (1979) 47-65.

[17] Q. Xiong, S. Aramideh, A. Passalacqua, S.-C. Kong, Characterizing effects of the shape of screw conveyors in gas-solid fluidized beds using advanced numerical models, Journal of Heat Transfer 137 (6) (2015) 061008.

[18] J. Xu, H. Qi, X. Fang, L. Lu, W. Ge, X. Wang, M. Xu, F. Chen, X. He, J. Li, Quasi-real-time simulation of rotating drum using discrete element method with parallel GPU computing, Particuology 9 (4) (2011) $446-450$.

[19] K. Chu, J. Chen, A. Yu, Applicability of a coarse-grained CFD-DEM model on dense medium cyclone, Minerals Engineering 90 (2016) 43-54.

[20] A. Munjiza, P. W. Cleary, Industrial particle flow modelling using discrete element method, Engineering Computations 26 (6) (2009) 698-743.

[21] K.-i. Tanida, K. Honda, N. Kawano, T. Kawaguchi, T. Tanaka, Y. Tsuji, Particle motion in screw feeder simulated by discrete element method, in: NIP \& Digital Fabrication Conference, Vol. 1998, Society for Imaging Science and Technology, 1998, pp. 429-431.

[22] M. Schutyser, W. Briels, A. Rinzema, R. Boom, Numerical simulation and PEPT measurements of a 3D conical helical-blade mixer: A high potential solids mixer for solid-state fermentation, Biotechnology 
and Bioengineering 84 (1) (2003) 29-39.

[23] A. Hassanpour, H. Tan, A. Bayly, P. Gopalkrishnan, B. Ng, M. Ghadiri, Analysis of particle motion in a paddle mixer using discrete element method (DEM), Powder Technology 206 (1) (2011) 189-194.

[24] M. Alian, F. Ein-Mozaffari, S. R. Upreti, Analysis of the mixing of solid particles in a plowshare mixer via discrete element method (DEM), Powder Technology 274 (2015) 77-87.

[25] M. Sakai, Y. Shigeto, G. Basinskas, A. Hosokawa, M. Fuji, Discrete element simulation for the evaluation of solid mixing in an industrial blender, Chemical Engineering Journal 279 (2015) 821-839.

[26] G. Basinskas, M. Sakai, Numerical study of the mixing efficiency of a ribbon mixer using the discrete element method, Powder Technology 287 (2016) 380-394.

[27] Y. Gao, F. J. Muzzio, M. G. Ierapetritou, Optimizing continuous powder mixing processes using periodic section modeling, Chemical Engineering Science 80 (2012) 70-80.

[28] A. Dubey, A. Sarkar, M. Ierapetritou, C. R. Wassgren, F. J. Muzzio, Computational approaches for studying the granular dynamics of continuous blending processes, 1-DEM based methods, Macromolecular Materials and Engineering 296 (3-4) (2011) 290-307.

[29] P. Owen, P. Cleary, Prediction of screw conveyor performance using the discrete element method (DEM), Powder Technology 193 (3) (2009) 274-288.

[30] P. J. Owen, P. W. Cleary, Screw conveyor performance: comparison of discrete element modelling with laboratory experiments, Progress in Computational Fluid Dynamics, An International Journal 10 (5-6) (2010) 327-333.

[31] D. Kretz, S. Callau-Monje, M. Hitschler, A. Hien, M. Raedle, J. Hesser, Discrete element method (DEM) simulation and validation of a screw feeder system, Powder Technology 287 (2016) 131-138.

[32] L. Pezo, A. Jovanović, M. Pezo, R. Čolović, B. Lončar, Modified screw conveyor-mixers-discrete element modeling approach, Advanced Powder Technology 26 (5) (2015) 1391-1399.

[33] Q. Hou, K. Dong, A. Yu, DEM study of the flow of cohesive particles in a screw feeder, Powder Technology 256 (2014) 529-539.

[34] T. A. Kingston, Granular mixing visualization and quantification in a double screw mixer, Master's thesis, Iowa State University, Ames, Iowa, USA (2013).

[35] A. H. Mahmoudi, X. Besseron, F. Hoffmann, M. Markovic, B. Peters, Modeling of the biomass combustion on a forward acting grate using XDEM, Chemical Engineering Science 142 (2016) 32-41.

[36] T. Oschmann, M. Schiemann, H. Kruggel-Emden, Development and verification of a resolved 3D inner particle heat transfer model for the discrete element method (DEM), Powder Technology 291 (2016) 392-407.

[37] C. Kloss, C. Goniva, A. Hager, S. Amberger, S. Pirker, Models, algorithms and validation for opensource DEM and CFD-DEM, Progress in Computational Fluid Dynamics, An International Journal 12 (2-3) (2012) 140-152.

[38] H. Zhu, Z. Zhou, R. Yang, A. Yu, Discrete particle simulation of particulate systems: Theoretical developments, Chemical Engineering Science 62 (13) (2007) 3378-3396.

[39] H. Hertz, Über die berührung fester elastischer körper., Journal für die reine und angewandte Mathematik 92 (1882) 156-171.

[40] R. D. Mindlin, H. Deresiewica, Elastic spheres in contact under varying oblique forces, Journal of Applied Mechanics 20 (1953) 327-344.

[41] G. Chandratilleke, A. Yu, J. Bridgwater, K. Shinohara, A particle-scale index in the quantification of mixing of particles, AIChE Journal 58 (4) (2012) 1099-1118.

[42] L. Fan, J. Too, R. Rubison, F. Lai, Studies on multicomponent solids mixing and mixtures Part III. Mixing indices, Powder Technology 24 (1) (1979) 73-89.

[43] M. Arntz, W. Den Otter, W. Briels, P. Bussmann, H. Beeftink, R. Boom, Granular mixing and segregation in a horizontal rotating drum: A simulation study on the impact of rotational speed and fill level, AIChE Journal 54 (12) (2008) 3133-3146.

[44] M. Kwapinska, G. Saage, E. Tsotsas, Mixing of particles in rotary drums: A comparison of discrete element simulations with experimental results and penetration models for thermal processes, Powder Technology 161 (1) (2006) 69-78. 
Table 1: Simulation design for evaluating parameter effects on double-screw mixing performance.

\begin{tabular}{ccccc}
\hline Cases & $\begin{array}{c}\text { Rotation } \\
\text { speed } \omega \\
(\mathrm{RPM})\end{array}$ & $\begin{array}{c}\text { Flight } \\
\text { pitch } P / D\end{array}$ & $\begin{array}{c}\text { Biomass feed } \\
\text { rate } f(\mathrm{~kg} / \mathrm{h})\end{array}$ & Mixture system \\
\hline $1-1$ & 20 & 1.25 & 1.75 & \\
$1-2$ & 40 & 1.25 & 3.50 & Multicomponent \\
$1-3$ & 60 & 1.25 & 5.00 & \\
\hline $2-1$ & 40 & 0.75 & 2.00 & \\
$2-2$ & 40 & 1.25 & 3.50 & Multicomponent \\
$2-3$ & 40 & 1.75 & 4.00 & \\
\hline $3-1$ & 40 & 1.25 & 1.00 & \\
$3-2$ & 40 & 1.25 & 2.00 & Multicomponent \\
$3-3$ & 40 & 1.25 & 3.50 & Binary, $D_{b}=1.0 \mathrm{~mm}$, \\
\hline $4-1$ & 40 & 1.25 & 3.50 & $\begin{array}{c}D_{g}=1.0 \mathrm{~mm} \\
\text { 4-2 }\end{array}$ \\
\hline & 40 & 1.25 & 3.50 & $\begin{array}{c}\text { Binary, } D_{b}=2.0 \mathrm{~mm}, \\
D_{g}=1.0 \mathrm{~mm}\end{array}$ \\
\hline
\end{tabular}

Case 1-1, 1-2 and 1-3 are designed for the screw rotation speed study; case 2-1, 2-2 and 2-3 for the pitch length study; case 3-1, 3-2 and 3-3 for the biomass feed rate study; and case $4-1$ and $4-2$ for the mixture system study. 
Table 2: Models for calculating contact forces and torques in DEM.

Force or torque

Equation

\begin{tabular}{|c|}
\hline $\begin{array}{c}-\frac{4}{3} E^{*} \sqrt{R^{*}} \delta_{n}^{3 / 2} \hat{\boldsymbol{n}}_{i j}+ \\
\sqrt{\frac{20}{3}} \beta\left(m^{*} E^{*} \sqrt{R^{*} \delta_{n}}\right)^{1 / 2} \boldsymbol{v}_{n, i j}\end{array}$ \\
\hline $\begin{array}{c}-8 G^{*} \sqrt{R^{*} \delta_{n}} \boldsymbol{\delta}_{t}+ \\
\sqrt{\frac{20}{3}} \beta\left(4 m^{*} G^{*} \sqrt{R^{*} \delta_{n}}\right)^{1 / 2} \boldsymbol{v}_{t, i j}\end{array}$ \\
\hline Torque $\boldsymbol{T}_{t, i j}^{c}$ \\
\hline$-\frac{4}{3} \mu_{r} E^{*} R^{*} \sqrt{R^{*}} \delta_{n}^{3 / 2} \boldsymbol{\omega}_{i j} /\left|\boldsymbol{\omega}_{i j}\right|$ \\
\hline $\begin{array}{l}\text { where, } \frac{1}{m *}=\frac{1}{m_{i}}+\frac{1}{m_{j}}, \frac{1}{R *}=\frac{1}{R_{i}}+\frac{1}{R_{j}}, \frac{1}{E *}=\frac{\left(1-\nu_{i}^{2}\right)}{E_{i}}+\frac{\left(1-\nu_{j}^{2}\right)}{E_{j}} \\
\frac{1}{G *}=\frac{2\left(2-\nu_{i}\right)\left(1+\nu_{i}\right)}{G_{i}}+\frac{2\left(2-\nu_{j}\right)\left(1+\nu_{j}\right)}{G_{j}}, \beta=\ln (\mathrm{e}) / \sqrt{\ln ^{2}(\mathrm{e})+\pi^{2}} \\
\hat{\boldsymbol{n}}_{i j}=\left(\boldsymbol{x}_{j}-\boldsymbol{x}_{i}\right) /\left|\boldsymbol{x}_{j}-\boldsymbol{x}_{i}\right|, \boldsymbol{v}_{i j}=\boldsymbol{v}_{i}-\boldsymbol{v}_{j}+\boldsymbol{\omega}_{i} \times \boldsymbol{R}_{i}-\boldsymbol{\omega}_{j} \times \boldsymbol{R}_{j}, \\
\boldsymbol{\omega}_{i j}=\boldsymbol{\omega}_{i}-\boldsymbol{\omega}_{j}, \boldsymbol{v}_{n, i j}=\left(\boldsymbol{v}_{i j} \cdot \hat{\boldsymbol{n}}_{i j}\right) \hat{\boldsymbol{n}}_{i j}, \boldsymbol{v}_{t, i j}=\left(\boldsymbol{v}_{i j} \times \hat{\boldsymbol{n}}_{i j}\right) \times \hat{\boldsymbol{n}}_{i j}, \\
\boldsymbol{R}_{i}=R_{i} \hat{\boldsymbol{n}}_{i j} \\
\text { The tangential overlap }\left|\boldsymbol{\delta}_{t}\right| \text { is truncated to fulfill }\left|\boldsymbol{F}_{t, i j}^{c}\right| \leq \\
\mu\left|\boldsymbol{F}_{n, i j}^{c}\right| .\end{array}$ \\
\hline
\end{tabular}


Table 3: Biomass and glass material properties in the discrete element model.

\begin{tabular}{cccc}
\hline Material & Red oak & Glass & $\begin{array}{c}\text { Acrylic } \\
\text { plastic }\end{array}$ \\
\hline Density $\rho\left(\mathrm{kg} / \mathrm{m}^{2}\right)$ & 550 & 2510 & - \\
\hline Diameter $D_{p}(\mathrm{~mm})^{\dagger}$ & $\begin{array}{c}1.0,1.5,2.5, \\
3.5,4.5,5.5\end{array}$ & 1.0 & - \\
\hline Young's Modulus $E(\mathrm{~Pa})$ & $2.6 \times 10^{6}$ & $2.6 \times 10^{6}$ & $2.6 \times 10^{6}$ \\
\hline Poisson's ratio $\nu$ & 0.29 & 0.25 & 0.4 \\
\hline Coefficient of restitution $e^{\ddagger}$ & 0.4 & 0.9 & 0.9 \\
\hline Coefficient of friction $\mu$ & & 0.2 & \\
\hline $\begin{array}{c}\text { Coefficient of rolling } \\
\text { friction } \mu_{r}\end{array}$ & & $1 \times 10^{-4}$ & \\
\hline
\end{tabular}

* Red oak is the material for biomass particle. Acrylic plastic is used for the mixer structure.

$\dagger$ Mass-based uniform distribution with equal fraction of each particle size.

$\ddagger$ This row shows the coefficient of restitution between the same material. The coefficient between red oak and glass is 0.4. The coefficient between red oak and acrylic plastic is 0.4 . The coefficient between glass and acrylic plastic is 0.9 . 
Figure 1. Geometry of the double screw mixer. Biomass particles are fed into the mixer at inlet 1 and glass beads are fed into the mixer at inlet 2. Mixer dimensions of mixing length $L$, width $W$, height $H$ and shaft center distance $S$ are fixed in this study. Screw dimensions of flight diameter $D$ and shaft diameter $d$ are also fixed, and screw pitch $P$ varies. Screws rotate in the opposite directions pumping the particle down towards the trough wall (Counter-Rotating Down Pumping). Design dimensions: $L=10 D=254 \mathrm{~mm}$, $W=50.19 \mathrm{~mm}, H=29.54 \mathrm{~mm}, S=21.59 \mathrm{~mm}$ and $d=3.97 \mathrm{~mm}$.

Figure 2. Grid system for local mixing analysis.

Figure 3. Visual comparison of experimental and simulated particulate flows. In the experimental snapshots, the color of biomass particles is brown and the glass beads are gray. In the simulation snapshots, blue represents biomass particles and red is used for glass beads. The operating conditions were: $w=40 \mathrm{RPM}, P / D=1.25$ and $f=3.5 \mathrm{~kg} / \mathrm{h}$ (case $1-2)$.

Figure 4. Biomass residence time in terms of screw rotation speed with non-dimensional pitch length of $P / D=1.25$. The same volumetric fill level was achieved by adjusting the biomass mass feed rate while maintaining a 1:10 biomass-to-glass bead mass flow ratio: $f=1.75 \mathrm{~kg} / \mathrm{h}$ for $\omega=20 \mathrm{RPM}, f=3.5 \mathrm{~kg} / \mathrm{h}$ for $\omega=40 \mathrm{RPM}$ and $f=5.0 \mathrm{~kg} / \mathrm{h}$ for $\omega=60$ RPM. Error bar shows the standard deviation of biomass particle residence time in the simulation. Experimental data came from [11, 34].

Figure 5. Biomass mean mixing time in terms of (a) screw rotation speed, (b) pitch length and (c) biomass mass feed rate. In (a) and (b), the volumetric fill level in the mixer remained constant. The error bar shows $95 \%$ confidence interval.

Figure 6. Illustration of two random particle pathlines in the mixer with operating conditions of $\omega=40 \mathrm{RPM}, P / D=1.25$ and $f=3.5 \mathrm{~kg} / \mathrm{h}$. The two particles are distinguished by different colors.

Figure 7. Snapshots of particle mixing at different screw rotation speeds with nondimensional pitch length $P / D=1.25$. The snapshots were taken at $L_{m}=3.75 D$ from the glass bead feed inlet. The volumetric fill level remained constant by adjusting the biomass mass feed rate while maintaining a 1:10 biomass-to-glass bead mass flow ratio. Blue is used to represent biomass particles and red for glass beads. Only particles in the right screw housing are shown. Five phases in a full rotation period are observed from top to bottom in each column.

Figure 8. Snapshots of particle mixing for different screw pitch lengths with screw rotation speed $\omega=40 \mathrm{RPM}$. The snapshots were taken at $L_{m}=3.75 \mathrm{D}$ from the glass bead feed inlet. The volumetric fill level remained constant by adjusting the solid mass feed rate while maintaining a 1:10 biomass-to-glass bead mass flow ratio. Blue is used to represent 
biomass particles and red for glass beads. Five phases in a full rotation period are observed from top to bottom in each column.

Figure 9. Snapshots of the particle distribution in the longitudinal section for different screw pitch lengths with screw rotation speed $\omega=40$ RPM. The snapshots were obtained by looking at the slice cut from right side. The volumetric fill level remained constant by adjusting the solid mass feed rate while maintaining a 1:10 biomass-to-glass bead mass flow ratio. Blue is used to represent biomass particles and red for glass beads.

Figure 10. Snapshots of particle mixing at different biomass feed rates while maintaining a 1:10 biomass-to-glass bead mass flow ratio with screw rotation speed $\omega=40 \mathrm{RPM}$ and non-dimensional pitch length $P / D=1.25$. The snapshots were taken at $L_{m}=3.75 D$ from the glass bead feed inlet. Blue is used to represent biomass particles and red for glass beads. Five phases in a full rotation period are observed from top to bottom in each column.

Figure 11. The mixing index at each sample plane in the axial direction. The gray dash line shows the glass feed inlet position.

Figure 12. Snapshots of particle mixing in different mixer systems with screw rotation speed $\omega=40$ RPM, non-dimensional pitch length $P / D=1.25$, and biomass feed rate $f=3.5 \mathrm{~kg} / \mathrm{h}$. The snapshots were taken at $L_{m}=3.75 \mathrm{D}$ from the glass bead feed inlet. Blue is used to represent biomass particles and red for glass beads. Five phases in a full rotation period are observed from top to bottom in each column. Binary mixture I: $D_{b}=1.0 \mathrm{~mm}$, $D_{g}=1.0 \mathrm{~mm}$; Binary mixture II: $D_{b}=2.0 \mathrm{~mm}, D_{g}=1.0 \mathrm{~mm}$; Multicomponent mixture: $D_{b}$ has a size distribution from 1.0 to $5.5 \mathrm{~mm}$ and $D_{g}=1.0 \mathrm{~mm}$.

Figure 13. The mixing index for instantaneous sampling at each plane for different mixtures. (a) Binary mixture: $D_{b}=1.0 \mathrm{~mm}, D_{g}=1.0 \mathrm{~mm}$. (b) Binary mixture: $D_{b}=$ $2.0 \mathrm{~mm}, D_{g}=1.0 \mathrm{~mm}$. (c) Multicomponent mixture: $D_{b}$ has a size distribution from 1.0 to $5.5 \mathrm{~mm}$ and $D_{g}=1.0 \mathrm{~mm}$. The operating conditions are constant for (a) and (b) as (c) except the mixture particle size. The gray dash line shows the glass feed inlet position. 

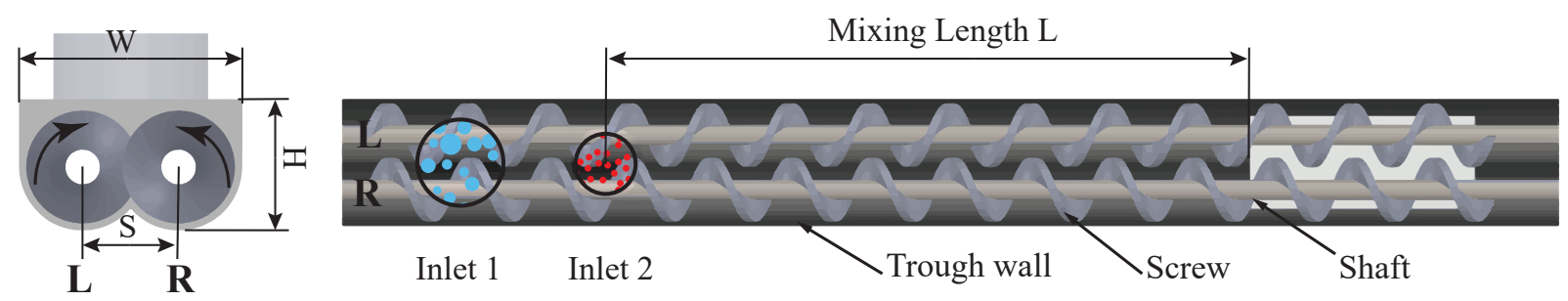

(a)

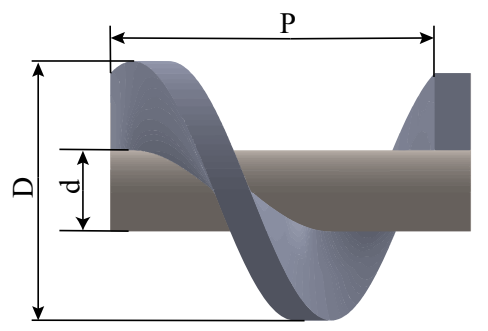

(b)

Figure 1: Geometry of the double screw mixer. Biomass particles are fed into the mixer at inlet 1 and glass beads are fed into the mixer at inlet 2. Mixer dimensions of mixing length $L$, width $W$, height $H$ and shaft center distance $S$ are fixed in this study. Screw dimensions of flight diameter $D$ and shaft diameter $d$ are also fixed, and screw pitch $P$ varies. Screws rotate in the opposite directions pumping the particle down towards the trough wall (Counter-Rotating Down Pumping). Design dimensions: $L=10 D=254 \mathrm{~mm}$, $W=50.19 \mathrm{~mm}, H=29.54 \mathrm{~mm}, S=21.59 \mathrm{~mm}$ and $d=3.97 \mathrm{~mm}$. 


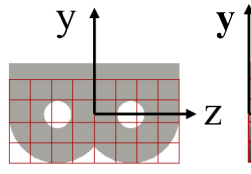

L $\quad \mathbf{R}$

Figure 2: Grid system for local mixing analysis. 


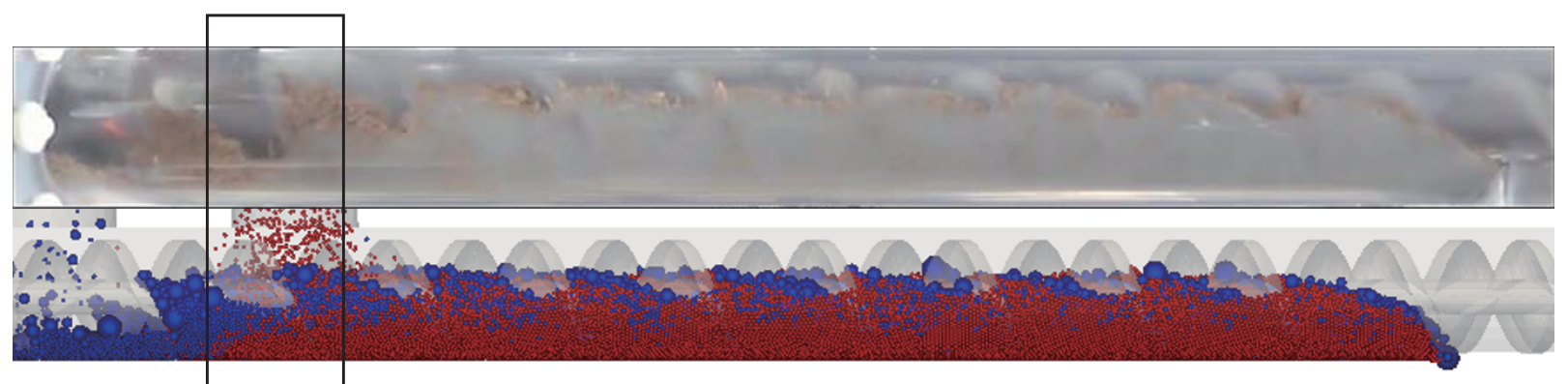

(a) Right view
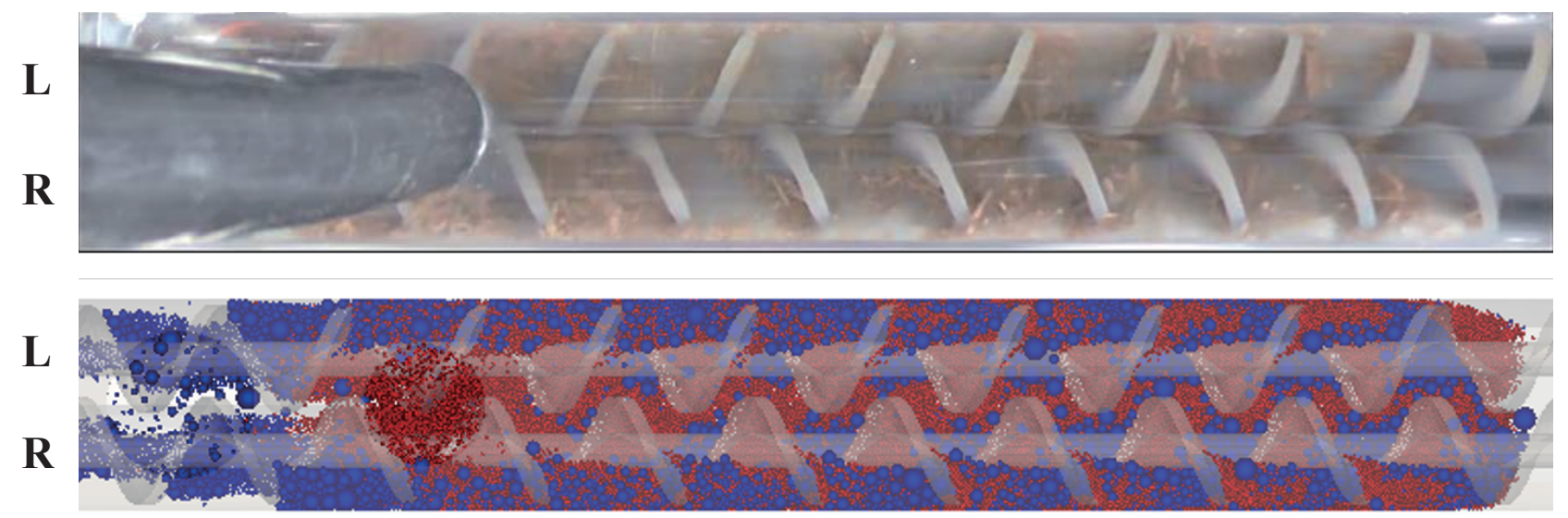

(b) Top view

Figure 3: Visual comparison of experimental and simulated particulate flows. In the experimental snapshots, the color of biomass particles is brown and the glass beads are gray. In the simulation snapshots, blue represents biomass particles and red is used for glass beads. The operating conditions were: $w=40 \mathrm{RPM}, P / D=1.25$ and $f=3.5 \mathrm{~kg} / \mathrm{h}$ (case $1-2$ ). 


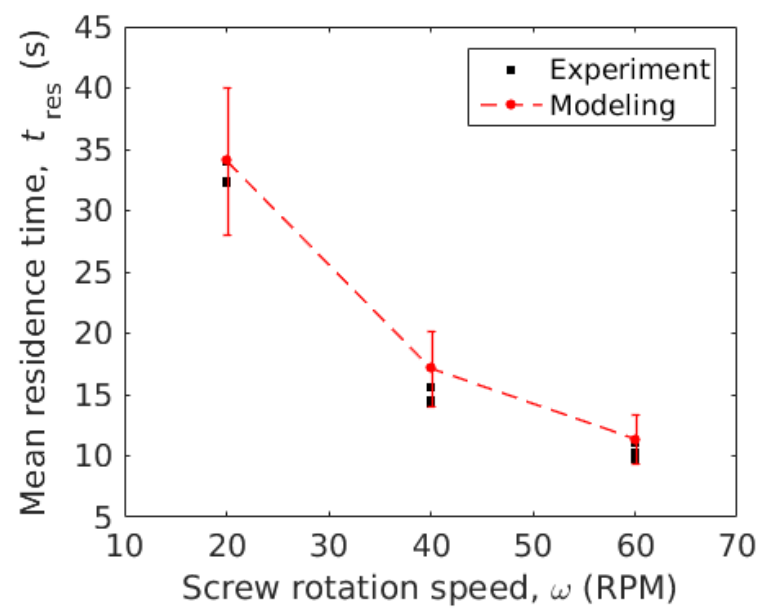

Figure 4: Biomass residence time in terms of screw rotation speed with non-dimensional pitch length of $P / D=1.25$. The same volumetric fill level was achieved by adjusting the biomass mass feed rate while maintaining a 1:10 biomass-to-glass bead mass flow ratio: $f=1.75 \mathrm{~kg} / \mathrm{h}$ for $\omega=20 \mathrm{RPM}, f=3.5 \mathrm{~kg} / \mathrm{h}$ for $\omega=40 \mathrm{RPM}$ and $f=5.0 \mathrm{~kg} / \mathrm{h}$ for $\omega=60$ RPM. Error bar shows the standard deviation of biomass particle residence time in the simulation. Experimental data came from [11,34]. 


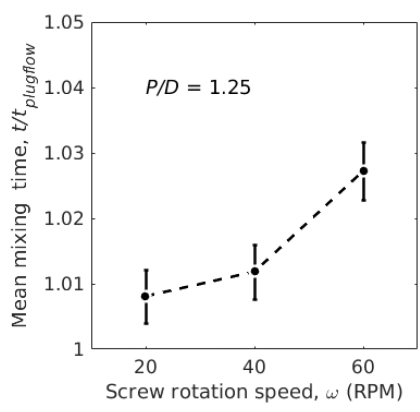

(a)

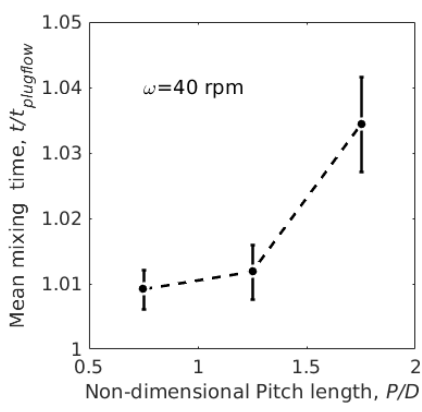

(b)

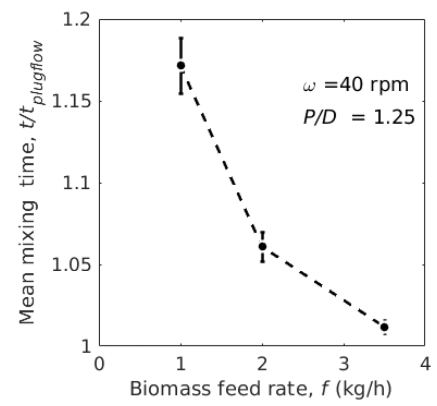

(c)

Figure 5: Biomass mean mixing time in terms of (a) screw rotation speed, (b) pitch length and (c) biomass mass feed rate. In (a) and (b), the volumetric fill level in the mixer remained constant. The error bar shows $95 \%$ confidence interval. 


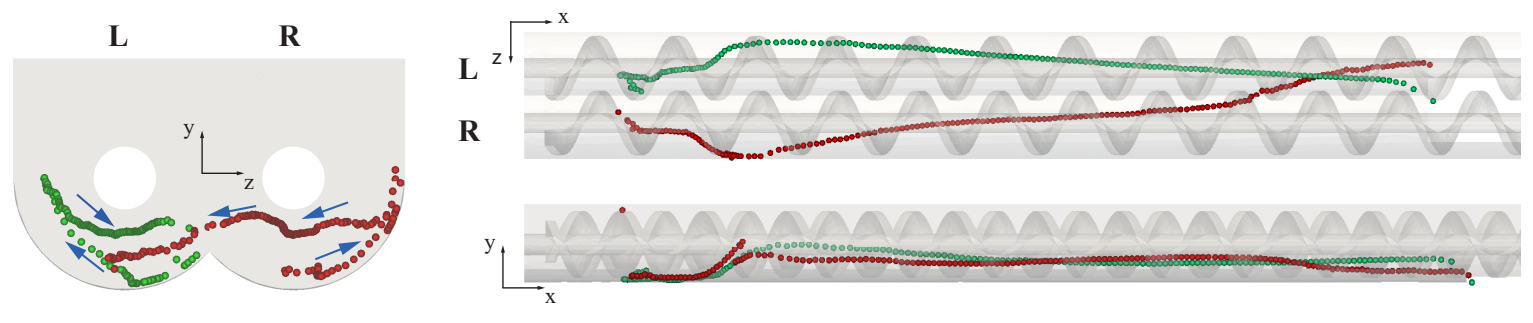

Figure 6: Illustration of two random particle pathlines in the mixer with operating conditions of $\omega=40 \mathrm{RPM}, P / D=1.25$ and $f=3.5 \mathrm{~kg} / \mathrm{h}$. The two particles are distinguished by different colors. 


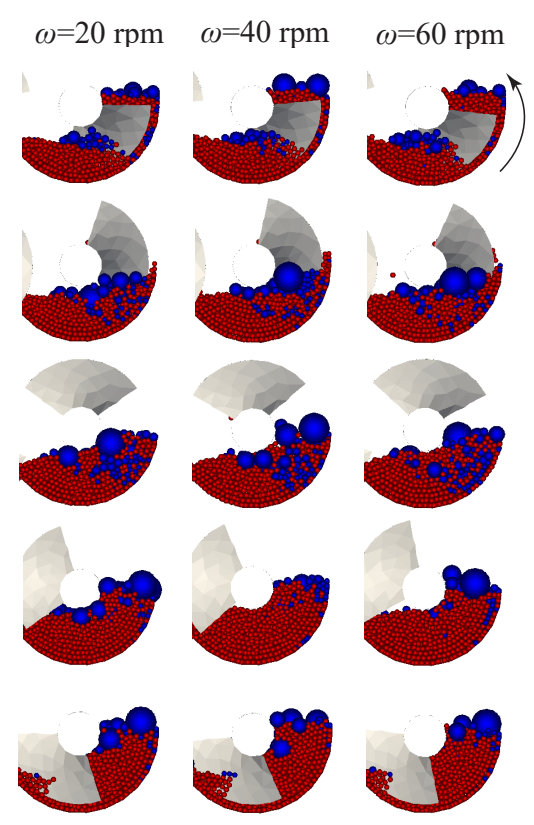

Figure 7: Snapshots of particle mixing at different screw rotation speeds with nondimensional pitch length $P / D=1.25$. The snapshots were taken at $L_{m}=3.75 D$ from the glass bead feed inlet. The volumetric fill level remained constant by adjusting the biomass mass feed rate while maintaining a 1:10 biomass-to-glass bead mass flow ratio. Blue is used to represent biomass particles and red for glass beads. Only particles in the right screw housing are shown. Five phases in a full rotation period are observed from top to bottom in each column. 


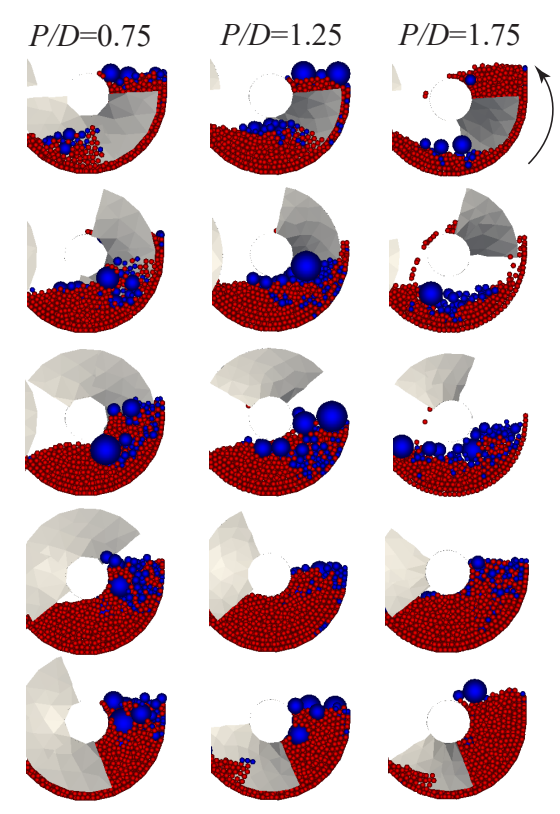

Figure 8: Snapshots of particle mixing for different screw pitch lengths with screw rotation speed $\omega=40$ RPM. The snapshots were taken at $L_{m}=3.75 D$ from the glass bead feed inlet. The volumetric fill level remained constant by adjusting the solid mass feed rate while maintaining a 1:10 biomass-to-glass bead mass flow ratio. Blue is used to represent biomass particles and red for glass beads. Five phases in a full rotation period are observed from top to bottom in each column. 


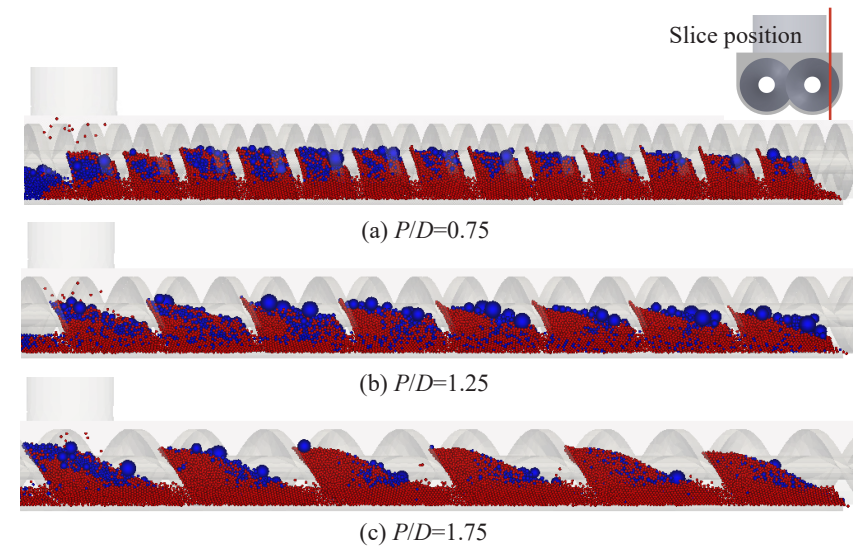

Figure 9: Snapshots of the particle distribution in the longitudinal section for different screw pitch lengths with screw rotation speed $\omega=40$ RPM. The snapshots were obtained by looking at the slice cut from right side. The volumetric fill level remained constant by adjusting the solid mass feed rate while maintaining a 1:10 biomass-to-glass bead mass flow ratio. Blue is used to represent biomass particles and red for glass beads. 


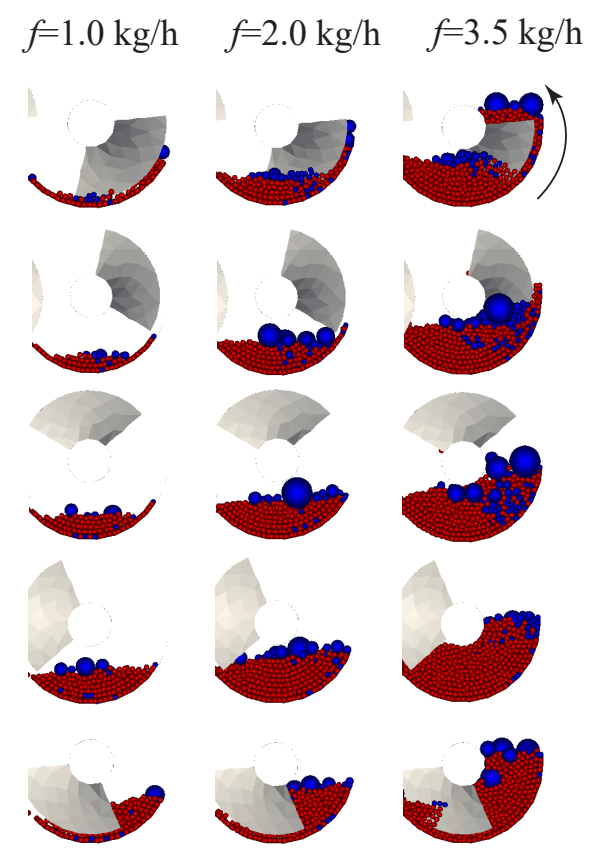

Figure 10: Snapshots of particle mixing at different biomass feed rates while maintaining a 1:10 biomass-to-glass bead mass flow ratio with screw rotation speed $\omega=40 \mathrm{RPM}$ and non-dimensional pitch length $P / D=1.25$. The snapshots were taken at $L_{m}=3.75 D$ from the glass bead feed inlet. Blue is used to represent biomass particles and red for glass beads. Five phases in a full rotation period are observed from top to bottom in each column. 


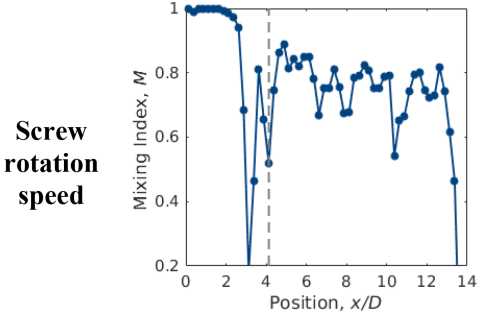

(a) $\omega=20 \mathrm{rpm}$
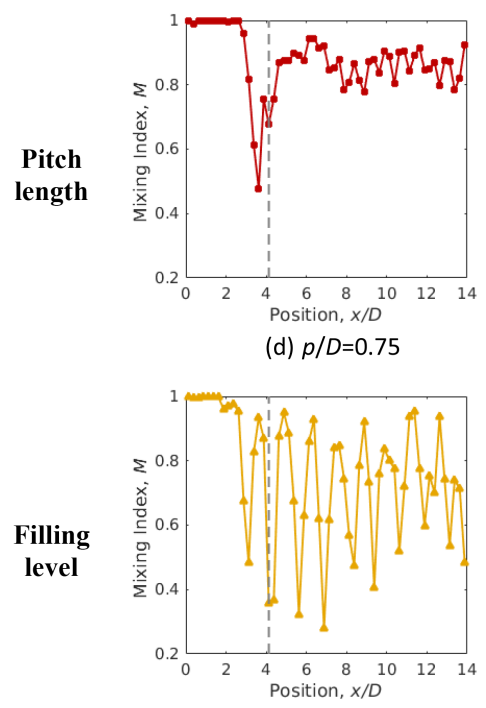

(g) $f=1.0 \mathrm{~kg} / \mathrm{h}$

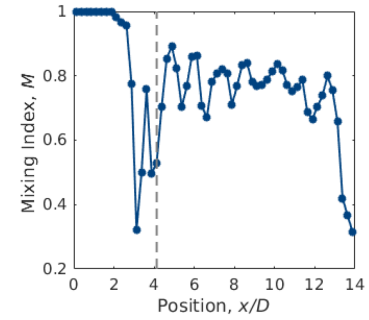

(b) $\omega=40 \mathrm{rpm}$
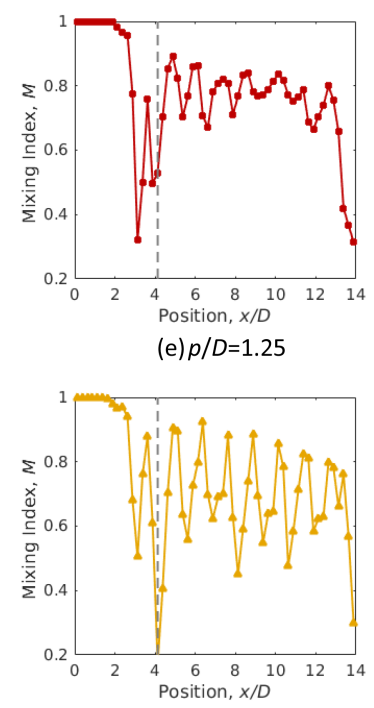

(h) $f=2.0 \mathrm{~kg} / \mathrm{h}$

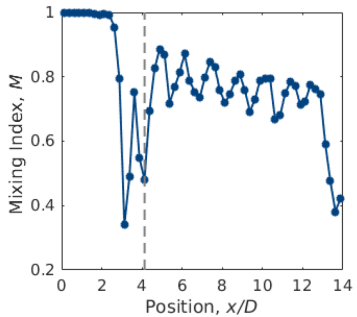

(c) $\omega=60 \mathrm{rpm}$
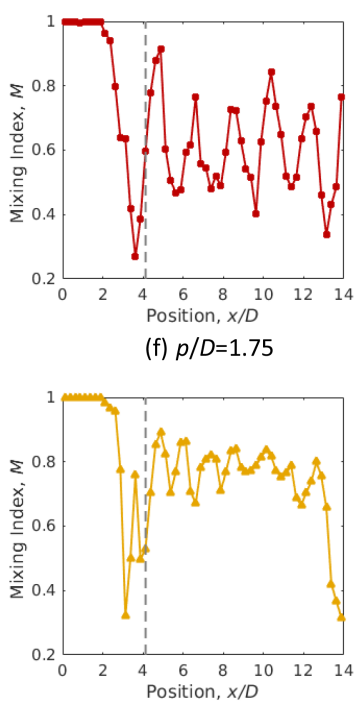

(i) $f=3.5 \mathrm{~kg} / \mathrm{h}$

Figure 11: The mixing index at each sample plane in the axial direction. The gray dash line shows the glass feed inlet position. 


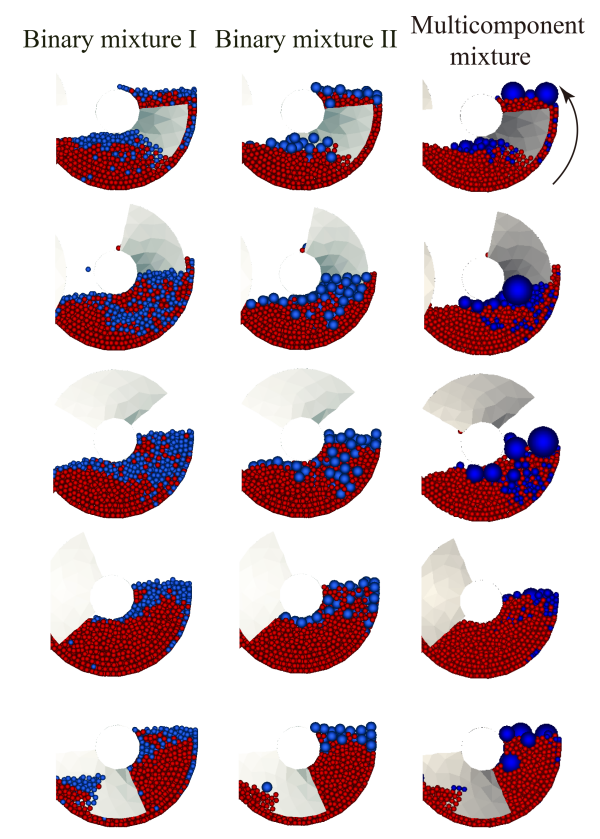

Figure 12: Snapshots of particle mixing in different mixer systems with screw rotation speed $\omega=40 \mathrm{RPM}$, non-dimensional pitch length $P / D=1.25$, and biomass feed rate $f=3.5 \mathrm{~kg} / \mathrm{h}$. The snapshots were taken at $L_{m}=3.75 \mathrm{D}$ from the glass bead feed inlet. Blue is used to represent biomass particles and red for glass beads. Five phases in a full rotation period are observed from top to bottom in each column. Binary mixture I: $D_{b}=1.0 \mathrm{~mm}$, $D_{g}=1.0 \mathrm{~mm}$; Binary mixture II: $D_{b}=2.0 \mathrm{~mm}, D_{g}=1.0 \mathrm{~mm}$; Multicomponent mixture: $D_{b}$ has a size distribution from 1.0 to $5.5 \mathrm{~mm}$ and $D_{g}=1.0 \mathrm{~mm}$. 


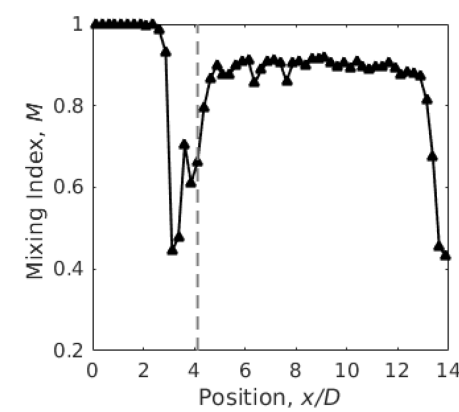

(a)

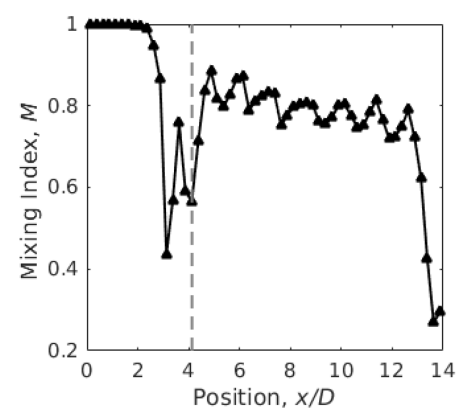

(b)

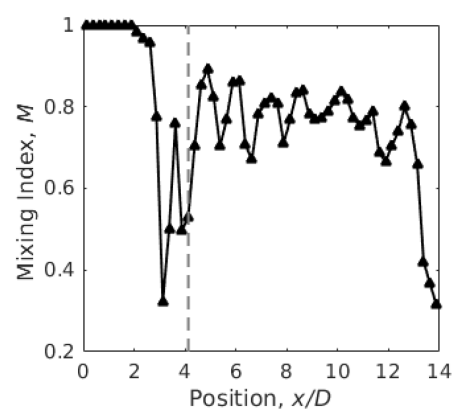

(c)

Figure 13: The mixing index for instantaneous sampling at each plane for different mixtures. (a) Binary mixture: $D_{b}=1.0 \mathrm{~mm}, D_{g}=1.0 \mathrm{~mm}$. (b) Binary mixture: $D_{b}=2.0 \mathrm{~mm}$, $D_{g}=1.0 \mathrm{~mm}$. (c) Multicomponent mixture: $D_{b}$ has a size distribution from 1.0 to $5.5 \mathrm{~mm}$ and $D_{g}=1.0 \mathrm{~mm}$. The operating conditions are constant for (a) and (b) as (c) except the mixture particle size. The gray dash line shows the glass feed inlet position. 


\section{Numerical study of particle mixing in a lab-scale screw mixer using the discrete element method}
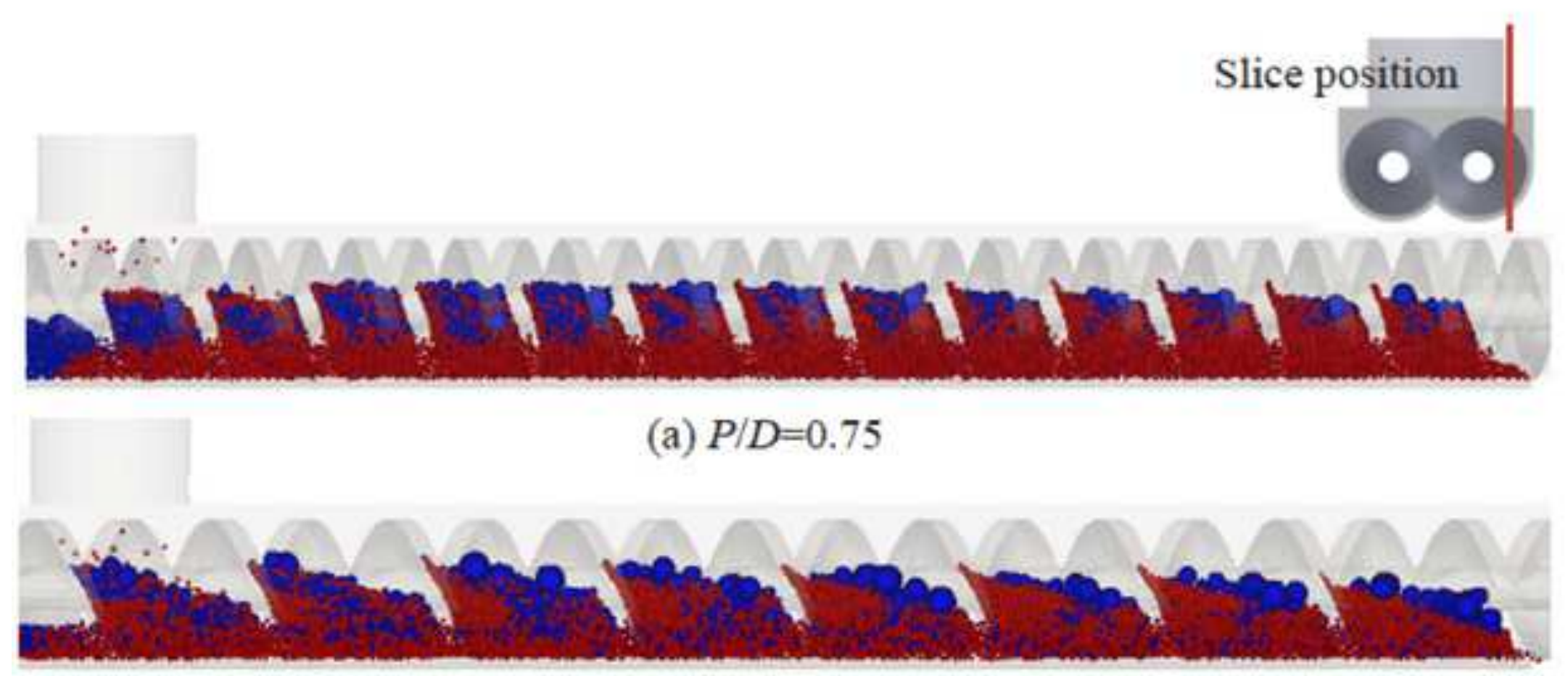

(b) $P / D=1.25$

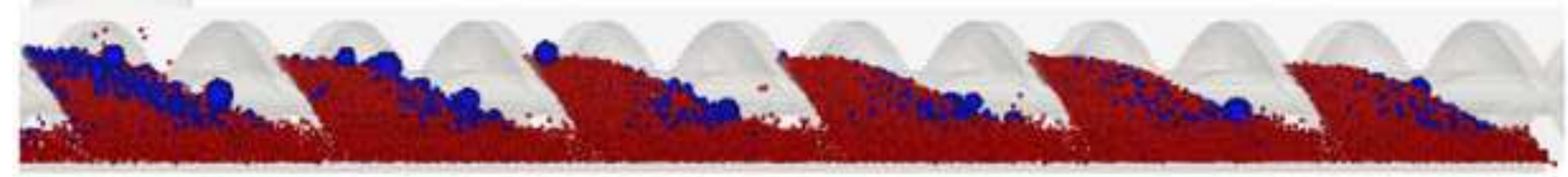

(c) $P / D=1.75$ 